\title{
Imaging features of central nervous system fungal infections
}

\author{
Krishan K. Jain, Shireesh K. Mittal, Sunil Kumar, Rakesh K. Gupta \\ Department of Radiology, Sanjay Gandhi Post-Graduate Institute of Medical Sciences, Lucknow - 226 014, India
}

\begin{abstract}
Fungal infections of the central nervous system (CNS) are rare in the general population and are invariably secondary to primary focus elsewhere, usually in the lung or intestine. Except for people with longstanding diabetes, they are most frequently encountered in immunocompromised patients such as those with acquired immunodeficiency syndrome or after organ transplantation. Due to the lack of inflammatory response, neuroradiological findings are often nonspecific and are frequently mistaken for tuberculous meningitis, pyogenic abscess or brain tumor. Intracranial fungal infections are being identified more frequently due to the increased incidence of AIDS patients, better radiological investigations, more sensitive microbiological techniques and better critical care of moribund patients. Although almost any fungus may cause encephalitis, cryptococcal meningoencephalitis is most frequently seen, followed by aspergillosis and candidiasis. The biology, epidemiology and imaging features of the common fungal infections of the CNS will be reviewed. The radiographic appearance alone is often not specific, but the combination of the appropriate clinical setting along with computed tomography or magnetic resonance may help to suggest the correct diagnosis.
\end{abstract}

Key words: Acquired immunodeficiency syndrome, brain, central nervous system, computed tomography, diffusion weighted imaging, fungal infection, infection, magnetic resonance (MR), proton MR spectroscopy

Fungal infections of the central nervous system (CNS) are rare and are invariably secondary to primary focus elsewhere, usually in the lung or intestine. Except for people with longstanding diabetes, these are most frequently encountered in immunocompromised patients such as those with acquired immunodeficiency syndrome (AIDS) or after organ transplantation. ${ }^{[1]}$ Due to the lack of inflammatory response, neuroradiological findings are often nonspecific and are frequently mistaken for tuberculous meningitis, pyogenic abscess or brain tumor. ${ }^{[1,2]}$ Intracranial fungal infections are being identified more frequently due to the increased incidence of AIDS, better radiological investigations, more sensitive microbiological techniques and better critical care of moribund patients. ${ }^{[2]}$ Although almost any fungus may cause encephalitis, cryptococcal meningoencephalitis is most frequently seen, followed by aspergillosis and candidiasis. ${ }^{[1]}$

The clinical and imaging features of the common fungal infections of the CNS will be reviewed. The radiographic appearance alone is usually nonspecific, however, when combined with appropriate clinical setting, may help in suggesting the correct diagnosis. ${ }^{[3]}$

\section{Cranial Infection}

\section{Aspergillosis}

Aspergillus is saprophytic opportunistic ubiquitous fungi found in soil, plants and grows as a mold on decaying vegetable matter. Aspergillus fumigatus is the most common human pathogen; however A. flavus, $A$. niger and $A$. oxyzae are also frequently seen. ${ }^{[2]}$ They have septate hyphae with dichotomous branching and produce spores. Humans are infected by inhaling these spores, with the lungs and paranasal sinuses as the primary site of infection. ${ }^{[4]}$

CNS aspergillosis is a rare condition with worldwide distribution. ${ }^{[4]}$ Most cases have been reported in adults, although the disease is also seen in children and neonates. ${ }^{[5]}$ Infection reaches the brain directly from the nasal sinuses or is hematogenous from the lungs and gastrointestinal tract. Rarely, the infection may contaminate the operative field during a neurosurgical procedure. ${ }^{[2]}$ In immunocompromised patients, aspergillosis usually occurs as part of a disseminated infection. ${ }^{[4]}$

The pathology of CNS aspergillosis can be classified into three forms: infarction, granulomas and meningitis. ${ }^{[5]}$ The fungal hyphae block intracerebral blood vessels, 
resulting in thrombosis and subsequent infarction and hemorrhage. ${ }^{[4,6]}$ The fungus can then spread beyond the vessel walls and form abscesses in the altered brain tissue. ${ }^{[6]}$ Purulent lesions may be chronic and have a tendency towards fibrosis and granuloma formation. ${ }^{[2]}$ Erosion of vessel wall can also form mycotic aneurysms. Aspergillosis is the most common cause of mycotic aneurysm. ${ }^{[6,7]}$ Microscopically, the most striking feature is the presence of the vascular invasion with thrombosis. In purulent lesions, pus is seen in the center of the abscesses with abundant polymorphs at the periphery. Granulomas consist of lymphocytes, plasma cells and fungal hyphae. ${ }^{[2]}$

The clinical diagnosis of cerebral aspergillosis is difficult because of nonspecific symptoms and fever may be absent. ${ }^{[5]}$ There may be clinical features of meningitis and subarachnoid hemorrhage. In patients with paranasal sinus disease orbital extension with proptosis, ocular palsies, visual deterioration and chemosis may occur. Aspergillosis should be considered in cases manifesting with acute onset of focal neurological deficits resulting from suspected vascular or space-occupying lesions, especially in immunocompromized hosts. ${ }^{[8-10]}$

The radiological appearance of Aspergillus infection of the CNS is variable and depends upon the immune status of the patients. Using computed tomography (CT) and magnetic resonance (MR), several patterns of cerebral aspergillosis have been reported: edematous lesions, hemorrhagic lesions, solid enhancing lesions referred to as aspergilloma or "tumoral form," [Figure 1] abscesslike ring-like enhancing lesions [Figure 2], infarction and mycotic aneurysm. ${ }^{[1]}$ Multiple areas of hypodensity on CT or hyperintensity on T2-weighted images (T2WI) on $\mathrm{MR}$, involving the cortex and/or subcortical white matter

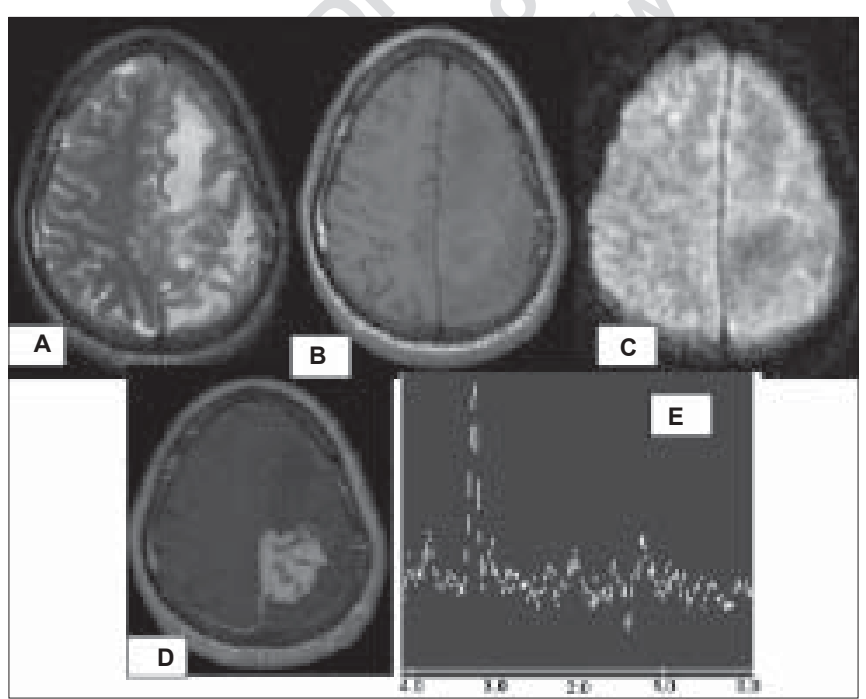

Figure 1: Aspergillus granuloma. Axial T2WI (A) showing a heterogeneous intensity lesion with surrounding hyperintensity in the left parietal region. Lesion is isointense on T1WI (B) and hypointense on DWI (C). Post-contrast image (D) shows intense enhancement of the mass lesion. PMRS (E) shows lactate (1.3 ppm) along with choline (3.2 ppm). Culture of the tissue was positive for Aspergillus flavus consistent with multiple areas of infarction is a common finding in Aspergillus infection. The superimposed hemorrhage may be identified as hyperdensity on CT and hyperintensity on T1-weighted images (T1WI) on MR. On MR, lesions may show areas of isointensity or low signal intensity on T2WI, which is attributed to fungal hyphae containing paramagnetic elements like manganese, iron and magnesium, but may also be related to blood

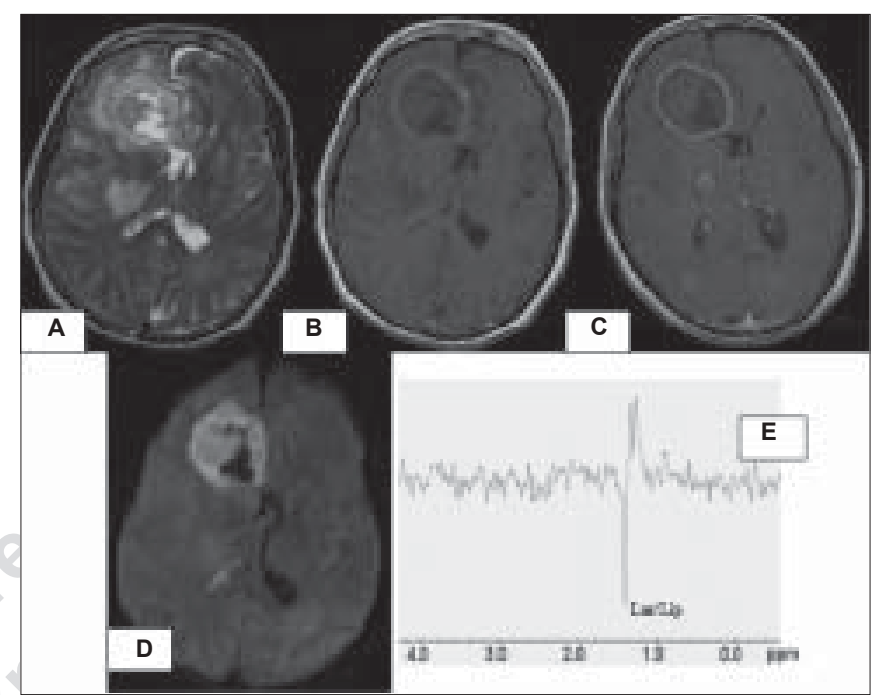

Figure 2: Fungal abscess in a 39-year-old immunocompromised male. Axial T2WI (A) shows a well-defined heterointense lesion with hypointense projections and wall in the right frontal lobe. Axial T1WI (B) shows a hypointense core with isointense intra-cavitary projections. Post-contrast axial T1WI(C) shows peripheral enhancement of wall with non-enhancing intra-cavitatory projections. Note the presence of two more enhancing lesions in the right thalamus and right occipital regions. DWI (D) shows hyperintensity in the projections with hypointensity in cavity. PMRS (E) shows lactate along with lipid (Lac/Lip,1.3ppm). Pus culture grew Aspergillus flavus
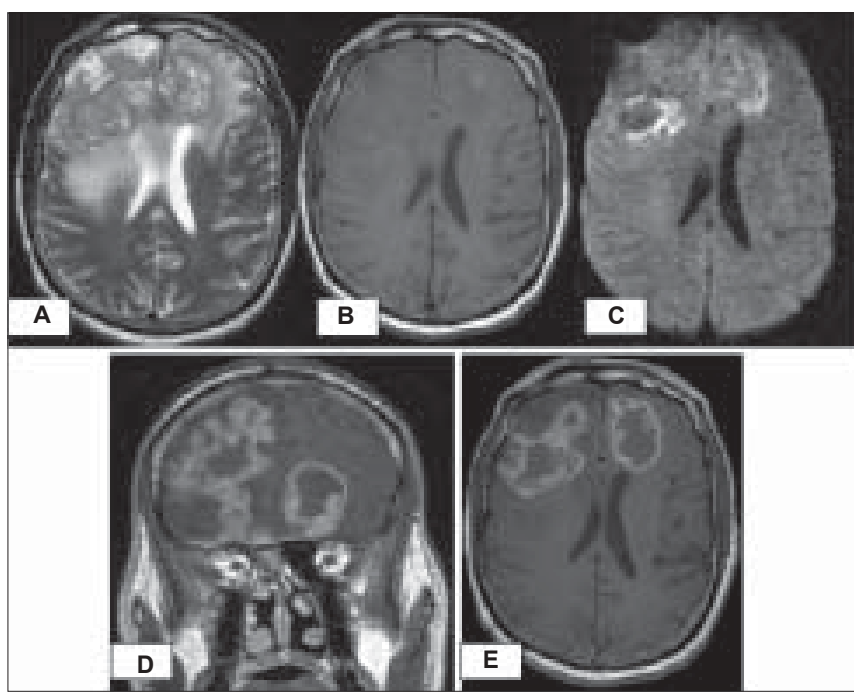

Figure 3: Rhinocerebral aspergillosis in an immunocompromised patient. Axial T2WI (A) showing heterogeneous intensity lesions with surrounding edema in bilateral frontal lobes. Lesions are iso to hyperintense on T1WI (B) and show mixed intensity on DWI (C). On post-contrast coronal (D) and axial (E) T1WI, lesions show heterogeneous peripheral enhancement with enhancing adjacent dura and involvement of ethmoid sinuses. Culture of the nasal scrapings grew Aspergillus flavus 
breakdown products. Dural enhancement is usually seen in lesions adjacent to infected paranasal sinuses, which represents direct extension of sinonasal disease [Figure 3]. Recognition of these radiological patterns in patients with cerebral aspergillosis is helpful in establishing an early diagnosis. ${ }^{[1,11]}$

Diffusion-weighted imaging (DWI) is valuable in early diagnosis of cerebral aspergillosis as it detects early infarction and can also be beneficial in differentiating these lesions from progressive multifocal leukoencephalopathy and neoplasm. ${ }^{[12]}$ Proton MR spectroscopy (PMRS) in an isolated case has shown elevation of glutamine-glutamate $(2.1 \mathrm{ppm})$, lactate (1.3 ppm) and amino acids (0.9 ppm) at the central non-enhancing part of the aspergillus abscess which is significantly different from the spectra of cystic or necrotic brain tumors. ${ }^{[13]}$ Proton MR Spectroscopy in aspergilloma is nonspecific with high choline, low creatine and lactate with no $\mathrm{N}$-acetyl aspartate (NAA) [Figure 1]..$^{[5]}$

Interestingly, lesions in perforating artery territories are more common in hematogenously disseminated aspergillosis involving the brain. This affinity for perforating artery is possibly related to the invasion of the walls of the involved larger arteries, subsequently compromising the origins of the perforating arteries. ${ }^{[14]}$

The CT appearance of aspergillosis sinusitis has been described as hypodense mass lesion with calcification and is separate from the wall of sinuses. In most cases, the adjacent bony structures show areas of erosion or sclerosis. ${ }^{[15]}$ On MR, Aspergillus sinusitis appears as iso to hypointense on T1WI and hypointense (may be mistaken for air in the sinuses) on T2WI. This T2 shortening is due to by presence of iron, magnesium, as well as increased calcium contents. ${ }^{[5,15]}$

\section{Cryptococcosis}

Cryptococcus neoformans, an encapsulated yeast-like fungus is the most common mycotic agent to affect the CNS. It is a ubiquitous organism found in mammal and bird feces, particularly in pigeon droppings. It causes disease primarily in patients with impaired immunity, particularly in those with AIDS. ${ }^{[16,17]}$ However, up to 30\% of the patients have been reported with no predisposing condition. ${ }^{[18]}$ Men are more commonly infected than women by cryptococcal infection. ${ }^{[5]}$

The infection is acquired through inhalation and spreads hematogenously to the CNS. The pathogenesis is remarkably similar to that of tuberculous meningitis. ${ }^{[19,20]}$ The central nervous system is the preferred site for cryptococcal infection, because soluble anticryptococcal factors present in serum are absent in cerebrospinal fluid (CSF) and the polysaccharide capsule of the fungus protects it from host inflammatory response. ${ }^{[16]}$

CNS infection can be either meningeal or parenchymal. ${ }^{[21]}$ Meningitis is often the primary manifestation and is most pronounced at the base of the brain. Parenchymal involvement is seen as cryptococcomas, dilated Virchow-Robin spaces or enhancing cortical nodules. It is believed that the meningeal infection along the base of the skull may involve the adjacent brain parenchyma, giving rise to cryptococcomas or may extend along the Virchow-Robin spaces. The commonest parenchymal sites are the midbrain and the basal ganglia. The pseudocysts are formed due to dilatation of the Virchow-Robin spaces with mucoid gelatinous material produced by the capsule of the organism. ${ }^{[17,21]}$ The term cryptococcoma should be used for lesions in which the fungus has invaded the parenchyma, producing chronic granuloma composed of macrophages, lymphocytes and foreign body giant cells.. ${ }^{[20]}$ They may also arise within the choroid plexus resulting in obstructive hydrocephalus. ${ }^{[19]}$ Cerebral infarctions, usually located in the basal ganglia, internal capsule and thalamus, may occur in $4 \%$ of patients with cryptococcal meningitis in the acute stage and during the treatment. ${ }^{[18]}$

Most patients with CNS cryptococcosis present with symptoms and signs of subacute meningitis or meningoencephalitis. Clinically, headache is the most common and sometimes the sole symptom. Meningeal features like photophobia and neck stiffness are uncommon. Other manifestations include symptoms and signs related to increased intracranial pressure due to hydrocephalus or related to encephalitis. ${ }^{[5,20]}$ Meningoencephalitis is associated with high morbidity and mortality, especially among immunocompromised hosts. ${ }^{[19,20]}$ Immunocompetent patients tend to present with localized, indolent neurological disease, more intense inflammatory responses but better clinical outcome. ${ }^{[22]}$

Cryptococcal disease may be diagnosed more readily than most other systemic fungal diseases for three reasons. The infection has a unique appearance on histopathology, the yeast can be seen in the CSF and the immunologic test for antigen in CSF and blood is highly sensitive and specific. ${ }^{[5]}$

The radiographic characteristics of CNS infection are protean and frequently minimal. ${ }^{[23]}$ The spectrum of MR and CT abnormalities ranges from no abnormality to meningeal enhancement, abscesses, intraventricular or intraparenchymal cryptococcomas, gelatinous pseudocysts and/or hydrocephalus. ${ }^{[16,23]}$

Hydrocephalus is the most common, although nonspecific finding. Intraparenchymal and intraventricular mass lesions are less common. ${ }^{[23]}$ Pseudocysts are seen as well-circumscribed, round to oval low-density lesions on CT and have CSF intensity on both T1WI and T2WI, which fail to enhance. ${ }^{[16]}$ Demonstration of clusters of these cysts in the basal ganglia [Figure 4] and thalami strongly suggest cryptococcal infection. ${ }^{[21]}$

Miliary lesions and cryptococcomas may present as variable density masses on CT and of low intensity on T1WI 


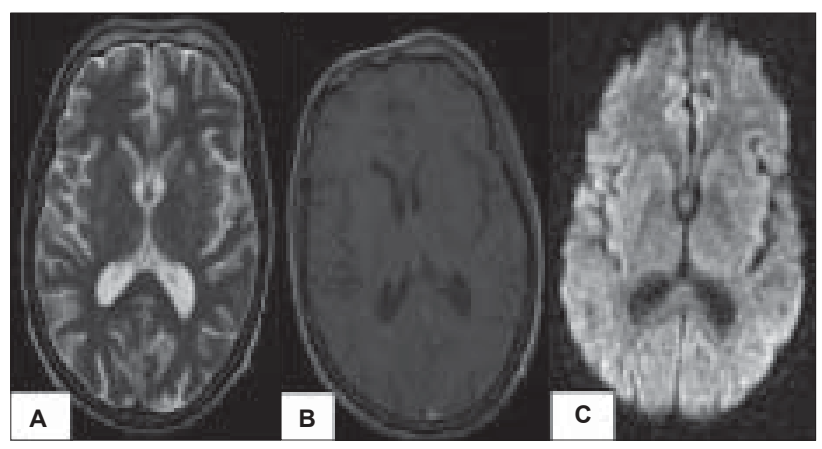

Figure 4: Cryptococcosis with dilated Virchow-Robin spaces in an immunocompromised patient. T2WI (A) shows multiple bilateral basal ganglia hyperintensities, which do not reveal any enhancement on postcontrast T1WI (B). Lesions show no restriction on DWI (C). CSF culture showed Cryptococcus neoformans

and high intensity on T2WI [Figure 5]. ${ }^{[23]}$ Granulomatous lesions are located preferentially on the ependyma of the choroid plexus and may enhance [Figure 4]. ${ }^{[16,23]}$ However, contrast enhancement of cryptococcomas or meninges is uncommon in immunocompromised patients due to the underlying immunosuppression and non-immunogenic nature of the polysaccharide capsule of the cryptococcal organism. ${ }^{[17,19,21]}$

Immunocompetent patients are more likely to present with cryptococcomas. ${ }^{[23]}$ Enhancement of these lesions might occur [Figure 6] as a result of an immunologic reaction by the host. ${ }^{[21]}$ Immediate and delayed imaging with a double dose of contrast has been reported to reduce the false negative studies by showing meningeal enhancement in immunocompromised patients. ${ }^{[24]}$

In vivo PMRS shows marked increase in lactate along with decrease in NAA, choline and creatine. ${ }^{[25]}$ Spectroscopy results suggest neuronal injury and gliosis in CNS cryptococcosis with decreased ratio of metabolite to nonsaturated water signal. ${ }^{[26]}$ Ex vivo PMRS has shown high concentration of alpha-trehalose and is considered as diagnostic of C. neoformans. ${ }^{[27]}$

On DWI, cryptococcoma shows hypointensity in the central cavity [Figure 5] and mimics a necrotic brain tumor, rather than a pyogenic brain abscess. ${ }^{[28]}$

\section{Mucormycosis}

Mucormycosis is a life-threatening opportunistic fungal infection caused by one of the members of the mucoraceal family. ${ }^{[29]}$ Rhizopus oryzae is the most common cause of infection. ${ }^{[30]}$ The hyphae are broad and non-septated, with right-angle branching and are distinct from Aspergillus. When spores are converted into hyphae, they become invasive, involve blood vessels and disseminate hematogenously or may spread through the paranasal sinuses into the brain and orbits. ${ }^{[31,32]}$

Diabetics comprise at least $70 \%$ of the reported cases and less than 5\% occur in normal hosts. ${ }^{[5]}$ Acidosis rather than hyperglycemia appears to be the important predisposing factor. ${ }^{[5,30]}$ Infection can also be seen

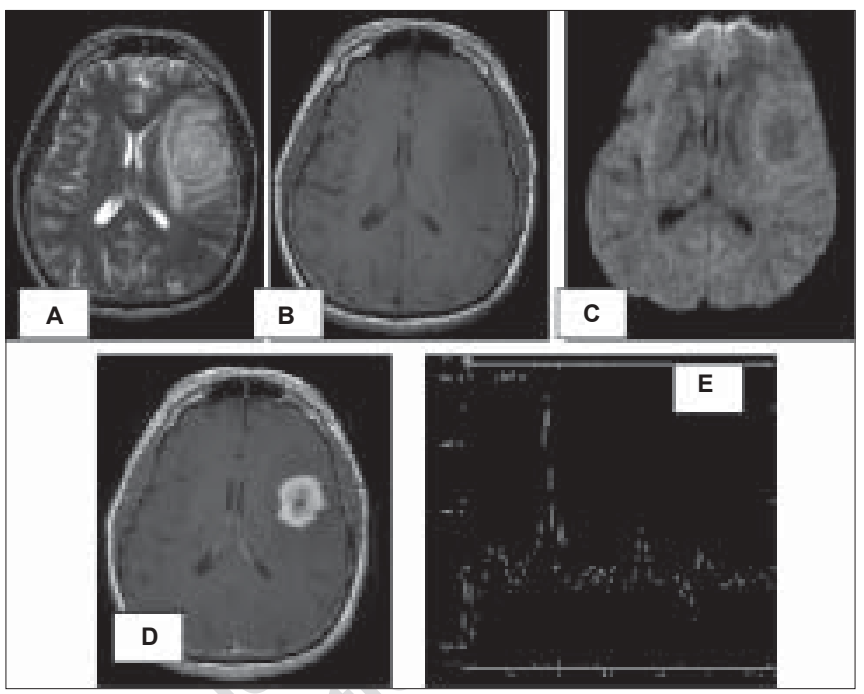

Figure 5: Cryptococcoma. Axial T2WI (A) showing a heterogeneous intensity lesion with surrounding hyperintensity in the left frontal region. Lesion is isointense on T1WI (B) and hypointense on DWI (C).

Post-contrast image (D) shows intense enhancement of the mass lesion. PMRS (E) shows lactate along with lipid (1.3 ppm), choline (3.2 ppm) and trehalose sugars at 3.8ppm. (Courtesy: Dr. CC Tchoyoson Lim, Department of Neuroradiology, National Neuroscience Institute, Singapore)

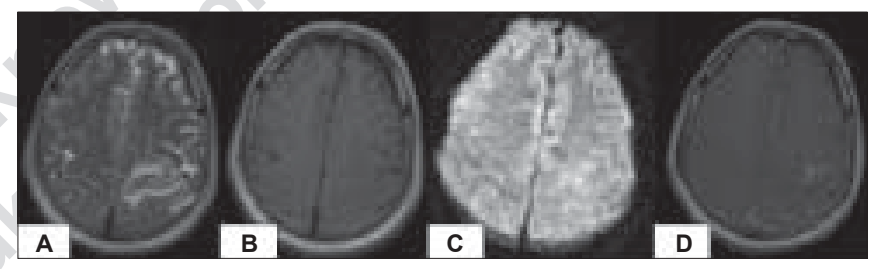

Figure 6: Cryptococcal meningoencephalitis in an immunocompetent patient. Axial T2WI (A) shows hyperintensity in the left parietal region. Lesion is hypointense on T1WI (B) and isointense on DWI (C). Postcontrast image (D) shows meningeal enhancement in the region of abnormality. CSF showed India ink positive yeast and culture confirmed it to be Cryptococcus neoformans

in intravenous drug abusers, in patients of anemia, leukemia, uremia and severe burns and in those receiving corticosteroid or chemotherapy. ${ }^{[33]}$

The rhinocerebral form is the most common infection. ${ }^{[29,30]}$ The organism may spread directly through the cribriform plate, via retrograde proliferation along the vessels or via extension into the orbit and then through the optic canal or superior orbital fissure into the cavernous sinus. ${ }^{[5]}$ The common presenting symptoms are headache, fever, sinusitis, facial swelling and unilateral orbital apex syndrome. ${ }^{[31]}$ Neurological deficits may result secondary to intracerebral abscess formation and thrombosis of major intracranial vessels. ${ }^{[29]}$ Prognosis is poor even after aggressive antifungal treatment and surgical debridement. ${ }^{[30]}$

Isolated CNS mucormycosis, a focal intracerebral infection, is rare and is mostly seen in drug abusers. It presents with acute onset and rapid development of neurological symptoms. The suspected source of infection is spores in the injected substances. Infarcts 
and abscesses are found on imaging studies, most commonly in the basal ganglia. ${ }^{[31,33]}$ A study reported involvement of basal ganglia in $82 \%$ of drug abusers while non-drug abusers showed involvement in only $9 \%$ of cases. ${ }^{[34]}$

Findings on CT and MR images include dense opacification of paranasal sinuses with variable mucosal thickening and usually absence of fluid levels in the maxillary, ethmoid, frontal and sphenoid sinuses, in decreasing order of incidence. Sinus contents have variable MR imaging characteristics, from hypointensity to hyperintensity on T2WI, probably secondary to the presence of manganese, iron and calcium. ${ }^{[29,31]}$ When present, the finding of bony erosion of the sinuses is strongly suggestive of the diagnosis in the appropriate clinical context. It is uncommon to visualize an organized retro-orbital mass. Although CT may sometimes show evidence of infection of the orbital soft tissue, MR is more sensitive. ${ }^{[30]}$

Intracranial findings include infarcts related to vascular thrombosis, mycotic emboli and frontal lobe abscesses [Figure 7]. ${ }^{[29]}$ On MR, involvement of brain parenchyma by the fungus can be separated from bland infarction due to vascular involvement. Contrastenhanced MR may help in distinguishing between these two possibilities. Involvement of cavernous sinuses, internal carotid and basilar artery in the form of abnormal vascular signal and abnormal enhancement has been shown secondary to thrombus formation in these vessels. ${ }^{[5]}$ Immunocompetent patients show different pattern of disease with absence of calcification and a predilection for the ethmoid sinuses. However, contrast enhancement, intraorbital and intracranial extension and high mortality rates are similar among all patients regardless of the status of their immune system. ${ }^{[31]}$

Siegal et al. reported elevated lactate, depleted NAA and presence of succinate and acetate in mucormycosis,

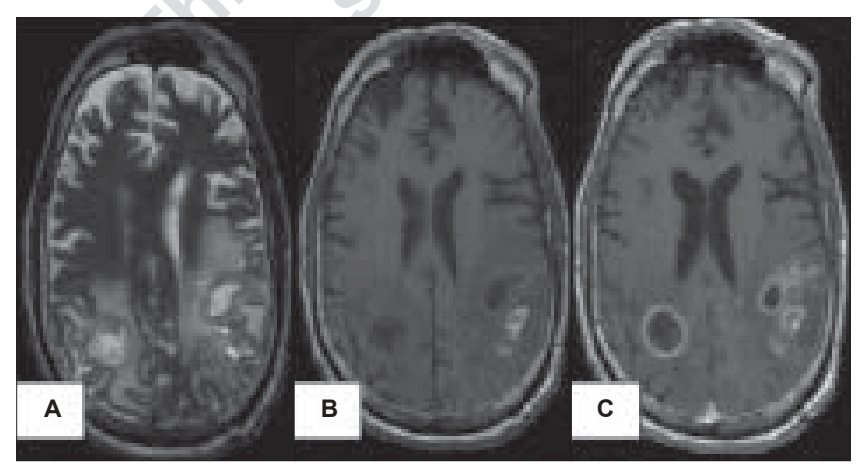

Figure 7: Multiple cerebral abscesses in a patient who had renal transplantation. Axial T2WI (A) at the level of ventricles showing multiple ill-defined hyperintense lesions with hypointense wall and surrounding edema in bilateral parietal region. Lesions are predominantly hypointense on T1WI (B) except a lesion in the left parietal region, which is hyperintense suggesting presence of hemorrhage within the lesion. Post-contrast T1WI (C) shows intense peripheral enhancement of these lesions. Pus culture grew Rhizopus oryzae without the commonly seen resonances of valine, leucine and isoleucine observed in bacterial abscess on PMRS. ${ }^{[35]}$

Diffusion restriction has been reported in a case of fungal cerebritis due to mucormycosis with apparent diffusion coefficient (ADC) values in the range of that reported for pyogenic brain abscess. ${ }^{[36]}$ In a case report of right-sided vision loss secondary to rhinocerebral mucormycosis, DWI demonstrated optic nerve infarction with restricted diffusion when regular MR was normal. Restricted diffusion may be the earliest detectable abnormality in acute ischemic optic neuropathy due to rhinocerebral mucormycosis. ${ }^{[37]}$

\section{Blastomycosis}

Blastomycosis, a thermally dimorphic fungus, is endemic in the southeastern and central United States. It is caused by Blastomyces dermatitidis. ${ }^{[38,39]}$ It is the yeast form that disseminates hematogenously, causing systemic disease. The fungus has been isolated from soil, which appears to be the reservoir for human infection. ${ }^{[5]}$

It is primarily a disease of the lung. Infection is usually acquired through inhalation of aerosolized conidia. ${ }^{[38,40]}$ Chronic pneumonia is the dominant clinical manifestation of blastomycosis, although extrapulmonary disease is also common, usually involving skin and subcutaneous tissue, bones and joints, prostate gland and CNS. ${ }^{[40]}$ Central nervous system infection is the result of either direct extension from the sinus or orbital infection or hematogenous spread. ${ }^{[5]}$ Central nervous system involvement occurs in $\sim 4 \%$ of patients with blastomycosis. ${ }^{[1]}$ It usually presents with meningitis, subdural focal leptomeningeal abscess or intraparenchymal abscess. ${ }^{[38]}$

It is surprising to know that blastomycosis does not have predilection for immunocompromised patients, as do other fungal organisms, such as Candida and cryptococcus. ${ }^{[42]}$ Although it is not an AIDS-defining infection, reports of blastomycosis in individuals infected with the human immunodeficiency virus (HIV) are increasing. ${ }^{[43]}$

Findings on CT and MR are nonspecific. CT shows the presence of an isodense or hyperdense lesion with surrounding edema, which demonstrates variable enhancement on contrast administration..$^{[5,42]}$ On MR, most granulomatous lesions are hyperintense on T2WI, however, dural lesions can be isointense or hypointense on both T1WI and T2WI. Contrast-enhanced study shows marked enhancement of granulomatous disease. ${ }^{[42]}$ Dural enhancement may also occur with direct extension from paranasal sinuses. ${ }^{[5]}$ The MR appearance of parenchymal blastomycosis abscess is indistinguishable from other causes of abscess. ${ }^{[5]}$ Isolating the organism is required for the diagnosis of blastomycosis as serologic tests are typically negative and are not useful..$^{[5,39]}$ 


\section{Coccidioidomycosis}

Coccidioidomycosis is a systemic infection caused by a dimorphic fungus Coccidiodes immitis. ${ }^{[4]}$ The organism is endemic to semiarid regions in the southwestern United States and northern Mexico. ${ }^{[45]}$ It exists in the mycelial form within the soil and becomes infective when the airborne arthrospores are inhaled. ${ }^{[4]}$ The heavier the inoculation, the more serious the resulting infection, as demonstrated by the increased incidence in endemic areas after severe dust storms or in members of archeologic expeditions who have disturbed the soil. ${ }^{[44,45]}$

Approximately, 4-5\% of symptomatic patients may develop disseminated disease with associated morbidity and mortality. ${ }^{[46,47]}$ Disseminated disease is even more common in immunocompromized patients. ${ }^{[5]}$ Central nervous system involvement is usually secondary to hematogenous dissemination from the lungs with meninges as the most common site. ${ }^{[48]}$

Radiological findings in patients of CNS coccidioidomycosis on imaging are related to: (1) meningitis and ependymitis; (2) hydrocephalus; (3) solitary or multiple granulomas; (4) white matter disease; (5) vasculitis; and (6) spinal arachnoiditis. ${ }^{[4]}$ Meningitis, a potentially lethal complication of coccidioidomycosis may be associated with cerebritis ${ }^{[5,49]}$ Hydrocephalus is seen in $68-93 \%$ of patients. ${ }^{[44,50]}$ Cerebral coccidioidal granulomas, either solitary or multiple, are less common than tuberculomas, but have been reported pathologically. ${ }^{[44]}$ Vasculitis has been observed in up to $40 \%$ of cases of meningitis; however, this typically involves the small penetrating branches of the major cerebral vessels, resulting in deep ischemic infarction. ${ }^{[48]}$ Ischemia has been reported in $58 \%$ of patients which may be related either to vasospasm or direct invasion of the vessel. ${ }^{[47,50]}$ Subarachnoid hemorrhage is a rare and fatal complication secondary to rupture of mycotic aneurysm. ${ }^{[51]}$

$\mathrm{MR}$ is superior to CT for detecting neuroimaging abnormalities in patients with coccidioidomycosis. ${ }^{[46]}$ Typically, diffuse and intense leptomeningeal enhancement of basilar, sylvian and interhemispheric cisterns is seen on post-contrast MR studies in coccidioidomycosis meningitis. ${ }^{[47,50]}$ Leptomeningeal enhancement can also extend to the spinal canal. Occlusion of the anterior spinal artery due to meningeal inflammatory process has also been reported..$^{[5,50]}$ Communicative or obstructive hydrocephalus develops after the granulomatous process about the fourth ventricle and basal meninges. ${ }^{[44]}$ Deep cerebral infarcts can be seen as areas of hyperintensity on T2WI. Less commonly, focal white matter or deep grey matter enhancing lesions representing granulomas are identified. Uncommonly, focal abscesses have been reported in HIV patients. ${ }^{[52]}$ Patients with documented neuroimaging abnormalities have a higher mortality. ${ }^{[46]}$

\section{Histoplasmosis}

Histoplasmosis, caused by a dimorphic fungus Histoplasma capsulatum, can range from subclinical infection to progressive disseminated disease. ${ }^{[53,54]}$ Infection is endemic in the upper Mississippi River and Ohio River valleys. ${ }^{[33]} \mathrm{H}$. capsulatum grows as a mold in the soil and causes infection when microconidia or hyphal elements are inhaled and convert into yeasts in the lungs or when organisms in old foci reactivate during immunosuppression. ${ }^{[39]}$ Disseminated disease is uncommon and occurs primarily when cellular defenses are impaired. ${ }^{[53]}$ Histoplasmosis represents the first manifestation of AIDS in $50-75 \%$ of patients. ${ }^{[39]}$

Clinical findings of progressive disseminated histoplasmosis are usually nonspecific. ${ }^{[53]}$ Clinical syndromes include subacute or chronic meningitis, focal brain or spinal cord lesions, stroke and diffuse encephalitis. ${ }^{[33,55]}$

CNS involvement is clinically recognized in $5-10 \%$ of cases of progressive disseminated histoplasmosis. However, in autopsy studies, it is reported in up to $25 \%$ of cases. ${ }^{[53,55]}$ Sites of CNS involvement include the basilar meninges, gray-white matter junction in the brain and rarely the spinal cord. ${ }^{[56]}$ Isolated involvement of the thalamus and choroid plexus has also been described. ${ }^{[5,57]}$ Based on pathological correlation CNS histoplasmosis has been described in three forms: Meningitis, Cerebritis and Vasculitis. ${ }^{[56]}$

Imaging features of CNS histoplasmosis are nonspecific. CT is abnormal in $~ 90 \%$ of cases, usually showing enhancing mass lesions, cerebral atrophy or hydrocephalus. ${ }^{[53]}$ On MR, the lesions are hyperintense on T2WI and hypointense on T1WI with perilesional edema and ring enhancement. In abscesses, the rim may be hypointense on T2WI due to the presence of paramagnetic free radicals in its wall. Diffuse leptomeningeal enhancement may also be present. ${ }^{[5]}$ Rarely, the disease may present as a histoplasmoma, which appears as an expansile lesion in the thalamic, hypothalamic and chiasmatic regions, with ring enhancement. ${ }^{[58]}$

\section{Candidiasis}

Candida species are ubiquitous yeasts, found on plants and as normal flora of the alimentary tract and mucocutaneous membranes of humans. ${ }^{[59]}$ Human candidiasis is most commonly caused by Candida albicans. The rapid multiplication in tissues, production of proteases, adhesions to extracellular matrix proteins and complement binding receptors are among the important factors contributing to its virulence. ${ }^{[60]}$

The clinical manifestations of candidiasis are primarily of three types: mucocutaneous, cutaneous and systemic or disseminated. Incidence of disseminated candidiasis is on the rise due to an increase in immunocompromised 
patients. Other risk factors include admission to intensive care units, total parenteral nutrition, multiple antibiotics, premature infants, corticosteroid use, chemotherapy and following organ transplantation, particularly during acute rejections. ${ }^{[61,62]}$

Primary candidiasis of the brain and meninges is rare; however, CNS invasion is reported in 18-52\% in disseminated candidiasis. ${ }^{[52,63]}$ Candida causes focal necrosis around the microcirculation mainly in the middle cerebral artery territory producing microabscesses. It can also cause vasculitis, intraparenchymal hemorrhage, aneurysms and thrombosis of small vessels with secondary infarction. ${ }^{[5,63]}$ Involvement of paranasal sinuses with extension to brain parenchyma with multiple abscess formation has also been reported [Figure 8]. ${ }^{[5]}$

CT usually underestimates the extent of the disease. Microabcesses appear iso to hypodense on nonenhanced CT and show multiple punctate enhancing nodules on contrast study. Granuloma may appear as hyperdense nodule on CT with nodular or ring enhancement. On MR, granuloma formation and brain abscess may have hypointense signals on T2WI due to the magnetic susceptibility effect of hemorrhage. Lesions show ring-enhancement on contrast administration. MR also shows features of associated meningitis, vasculitis and infarction. ${ }^{[5,63]}$

\section{Common MR features of brain abscess secondary to fungal infection}

Fungal abscesses are hypointense on T1WI and hyperintense on T2WI with well-defined rim enhancement on post-contrast images in immunocompetent patients. However, in immunocompromised patients, abscesses appear as patchy or punctate T2 hyperintense lesions with frequently absent enhancement. However, contrast

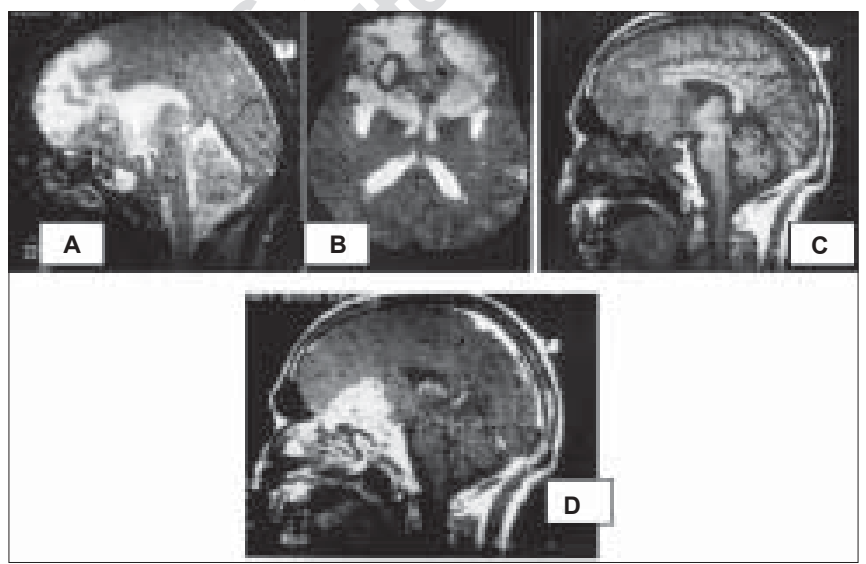

Figure 8: Rhinocerebral candidiasis. Sagittal T2WI (A) shows mixed intensity area in the basal frontal region with edema. Axial T2WI (B) shows multiple hyperintense areas with hypointense rims in the bilateral frontal lobes. Sagittal T1WI (C) shows the lesion to be isointense to gray matter. Post-contrast T1WI (D) shows heterogeneous enhancement of the lesion along with enhancing sphenoid and ethmoid sinuses. Pus culture from the brain grew Candida albicans enhanced imaging decreases the number of false negative studies. $^{[1,64,65]}$ Fungal lesions are known to show lipids (1.2-1.3 ppm), lactate (1.3 ppm), alanine (1.5 ppm), acetate (1.9 ppm), succinate (2.4 ppm), choline (3.2 ppm) and unidentified resonance at $3.8 \mathrm{ppm}$ on PMRS. ${ }^{[27,35,66]}$ In a recent study conducted in our institution fungal abscesses were differentiated from non-fungal lesions by combining the conventional, DWI and PMRS features. ${ }^{[67]} \mathrm{A}$ ring-enhancing, T2-heterointense lesion with irregular walls and non-enhancing intra-cavitary projections having low ADC carries a high probability of being a fungal abscess. These projections, directed centrally from the wall, are isointense to hypointense on T1WI and hypointense on T2WI [Figure 9]. The identification of multiple signals seen between 3.6 and $3.8 \mathrm{ppm}$ assigned to trehalose sugars on PMRS may further add to the diagnostic confidence. ${ }^{[67]}$

\section{Spinal Infections}

Fungal infections of the spine are relatively uncommon. They have been reported with Candida, aspergillosis, cryptococcus, coccidioidomycosis and histoplasmosis. Candida and Aspergillus produce disease when they gain access to the vascular system through intravenous lines, during implantation of prosthetic devices or during surgery. For the other fungi, spinal involvement usually is the result of hematogenous or direct spread of organisms from an initial pulmonary source of infection. ${ }^{[68]}$

The vertebral body, posterior bony vertebral elements,

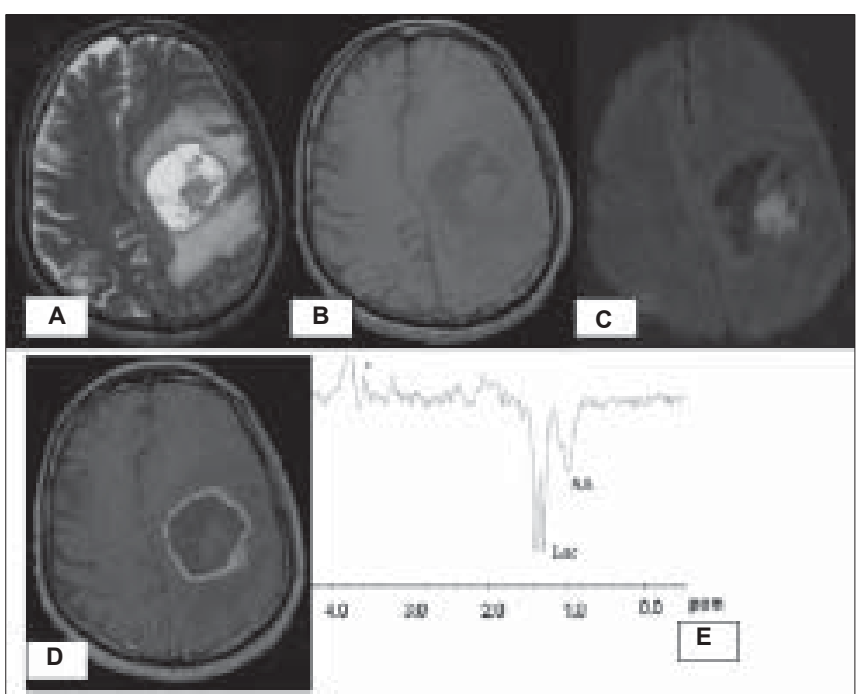

Figure 9: Fungal abscess in the left fronto-parietal region of a 46-year-old male immunocompetent patient. The lesion appears as a well-defined hyperintense mass with irregular wall and intra-cavitary hypointense projections on axial T2WI (A). Axial T1WI (B) shows hypointense core with isointense intra-cavitary projections. Post-contrast axial T1WI (C) shows peripheral enhancement of wall with no enhancement of intracavitary projections. Hyperintense projections with hypointense cavity are seen on DWI (D). PMRS (E) shows amino acids (AA, 0.9ppm) and lactate (Lac, 1.3ppm) with multiple peaks at 3.6 and $3.8 \mathrm{ppm}$. Pus culture grew Aspergillus flavus 
epidural space and perispinal soft tissues may be involved in the infectious process. Occasionally, the intervertebral disc may be relatively spared early in the disease. ${ }^{[69]}$

Fungal spondylitis secondary to Candida and Aspergillus is characterized by low signal intensity on T1WI and high signal intensity on T2WI with intervening disc involvement [Figure 10]. The bone marrow in the affected vertebral bodies may show low signal intensity on both T1WI and T2WI due to lack of inflammatory response in immunocompromised patients. ${ }^{[5,70]}$ In some cases, hyperintensity of the vertebral disc is absent and rarely, intranuclear cleft may be preserved. ${ }^{[71]}$ Epidural abscess may be seen in Aspergillus infection. There are reports of intradural abscess formation following epidural steroid injection in immunocompetent individuals. ${ }^{[5,72]}$

Skeletal coccidioidomycosis is frequently multicentric. The axial skeleton is the most common site. ${ }^{[73]}$ Spinal involvement is seen in approximately $25 \%$ of patients with disseminated disease. ${ }^{[7]}$ Spinal lesions are usually well-demarcated but may be ill-defined with permeative type of bone destruction. Plain radiographs are effective in the initial evaluation of bones and joints. CT and MR are useful in determining soft tissue involvement and spinal abnormalities. ${ }^{[73]}$ The typical imaging features include disc involvement, heterogeneous marrow signal alteration and extensive extra-osseous involvement with lack of bony deformity. ${ }^{[74}$ Other features include phlegmenous, enhancing non-liquefactive soft tissue abnormalities, cord compression and nerve root impingement. As the disease is multifocal, MR screening of the entire vertebral column often reveals occult areas of involvement. ${ }^{[74]}$ The extensive soft tissue and marrow involvement with lack of bony deformities are the features that help to differentiate coccidiomycosis

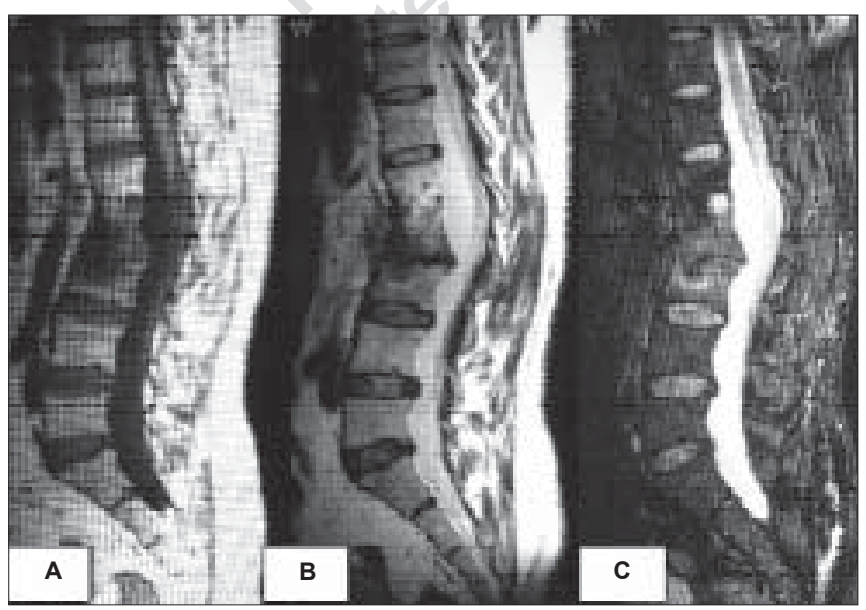

Figure 10: Aspergillus spondylitis. Sagittal T1WI (A) and T2WI (B) show collapse and partial fusion of first, second and third lumbar vertebral bodies with destruction of intervening disc. Fat saturated sagittal T2WI (C) showing active involvement with hyperintensity in the posterior part of the intervening disc between L1and L2 vertebrae from other causes of infective spondylitis. ${ }^{[5]}$ As the MR features are nonspecific, biopsy with culture is required to establish the diagnosis. ${ }^{[74]}$

Spinal cord disease is a rare presentation of cryptococcosis. ${ }^{[75]}$ Bony involvement is seen in $5 \%$ of disseminated cryptococcosis. ${ }^{[76]}$ Imaging findings are not specific and simulate spinal tuberculosis with involvement of the vertebral body along with posterior elements and paraspinous and perivertebral soft tissues with relative preservation of the disc. ${ }^{[77,78]}$ The vertebral body predominantly shows osteolytic lesions with discrete margins, absent surrounding sclerosis and periosteal reaction. ${ }^{[79]}$ Meningoradiculitis and spinal cryptococcal granulomas are rare [Figure 11]. ${ }^{[75,77]}$ In a case of spinal cryptococcus infection DWI shows hyperintensity of involved vertebral body, epidural space and posterior elements. ${ }^{[80]}$

Bone is the one of the frequent sites of disease in patients with blastomycosis, lower thoracic or lumbar vertebrae being most often affected. ${ }^{[81]} \mathrm{MR}$ reveals destructive vertebral changes, an epidural mass, psoas abscess and lack of involvement of the disc spaces. Sparing of the disc space is due to spread of infection by way of paravertebral structures and surrounding potential spaces. ${ }^{\left[{ }^{[2]}\right]}$ Blastomycosis can rarely present as an isolated intramedullary lesion. ${ }^{[83]}$

Treatment of fungal spondylitis is often delayed because of difficulty with the diagnosis that results in poor prognosis. Performing fungal cultures whenever a spinal infection is suspected might hasten the diagnosis. ${ }^{[84]}$

\section{References}

1. Kastrup O, Wanke I, Maschke M. Neuroimaging of infections. NeuroRx 2005;2:324-32.

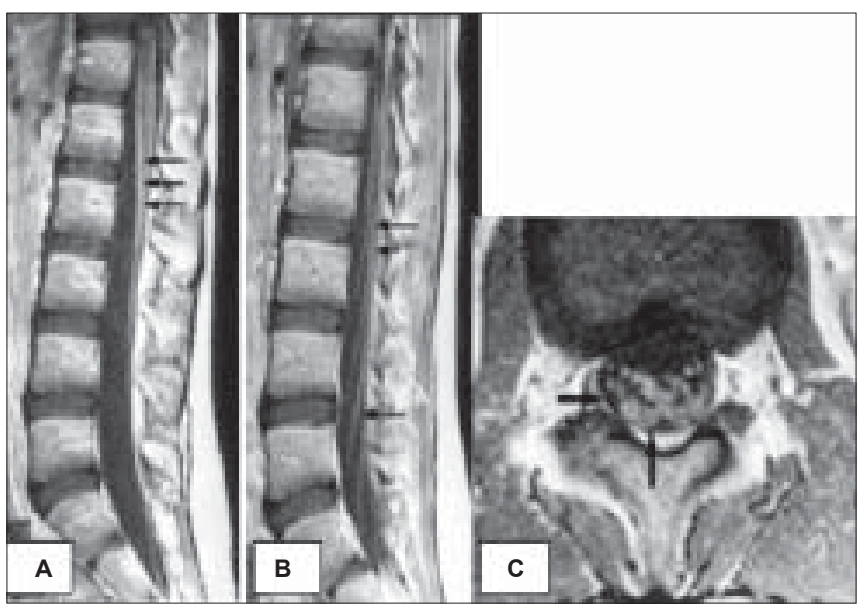

Figure 11: Cryptococcal meningoradiculitis. Post-contrast sagittal T1WI showing leptomeningeal enhancement $(A)$ and enhancement of cauda equina nerve roots (B). Axial T1WI (C) of cauda equina after injection of gadolinium showing massive nerve root enhancement. (Courtesy, Dr. Leonardo Deus-Silva, Medical Sciences School of the Campinas State University, Brazil) 
2. Nadkarni T, Goel A. Aspergilloma of the brain: An overview. J Postgrad Med 2005;51:37-41.

3. Ostrow TD, Hudgins PA. Magnetic resonance imaging of intracranial fungal infections. Top Magn Reson Imaging 1994;6:22-31.

4. Miaux Y, Ribaud P, Williams M, Guermazi A, Gluckman E, Brocheriou C, et al. MR of cerebral aspergillosis in patients who have had bone marrow transplantation. AJNR Am J Neuroradiol 1995;16:555-62.

5. Kathuria MK, Gupta RK. Fungal infections. In: Gupta RK, Lufkin RB, editors. MR imaging and spectroscopy of central nervous system infections. Kluwer Press: New York; 2001. p. 177-203.

6. Tempkin AD, Sobonya RE, Seeger JF, Oh ES. Cerebral aspergillosis: Radiologic and pathologic findings. Radiographics 2006;26:1239-42.

7. Walsh T.J, Hier DB, Caplan LR. Aspergillosis of central nervous system: Clinicopathological analysis of 17 patients. Ann Neurol 1985;18:57482.

8. Hall WA. Neurosurgical infections in the compromised host. In: Haines SJ, Hall WA, editors. Neurosurgery clinies of North America, Infections in neurologic surgery. Vol 3. WB Saunders Co: Philadelphia; 1992. p. $435-42$.

9. Saravia-Gomez J. Aspergillosis of the Central Nervous System. In: Vinken PJ, Bruyn GW, editors. Handbook of Clinical Neurology, Vol 35, NorthHolland Publishing Company: Holland; 1978. p. 395-400.

10. Sharma RR, Lad SD, Desai AP, Lynch PG. Surgical management of fungal infections of the nervous system. In: Schmidek HH, editor. Schmidek and sweet operative neurosurgical techniques: Indications, methods and results. $4^{\text {th }}$ ed. WB Saunders Company: Philadelphia; 2000. p. 1726-55

11. Ashdown BC, Tien RD, Felsberg GJ. Aspergillosis of the brain and paranasal sinuses in immunocompromized patients: CT and MR imaging findings. AJR Am J Roentgenol 1994;162:155-9.

12. Keyik B, Edgüer T, Hekimoğlu B. Conventional and diffusion-weighted MR imaging of cerebral aspergillosis. Diagn Interv Radiol 2005;11:199201.

13. Oner AY, Celik H, Akpek S, Tokgoz N. Central nervous system aspergillosis: Magnetic resonance imaging, diffusion-weighted imaging and magnetic resonance spectroscopy features. Acta Radiol 2006;47:40812 .

14. DeLone DR, Goldstein RA, Petermann G, Salamat MS, Miles JM, Knechtle SJ, et al. Disseminated aspergillosis involving the brain: Distribution and imaging characteristics. AJNR Am J Neuroradiol 1999;20:1597-604.

15. Chang T, Teng MM, Wang SF, Li WY, Cheng CC, Lirng JF. Aspergillosis of the paranasal sinuses. Neuroradiology 1992;34:520-3.

16. Kovoor JM, Mahadevan A, Narayan JP, Govindappa SS, Satishchandra P, Taly AV, et al. Cryptococeal choroid plexitis as a mass lesion: MR imaging and histopathologic correlation. AJNR Am J Neuroradiol $2002 ; 23: 273-6$

17. Awasthi M, Patankar T, Shah P, Castillo M. Cerebral cryptococcosis: Atypical appearances on CT. Br J Radiol 2001;74:83-5.

18. Aharon-Peretz J, Kliot D, Finkelstein R, Ben Hayun R, Yarnitsky D, Goldsher D. Cryptococcal meningitis mimicking vascular dementia. Neurology 2004;62:2135.

19. Sibtain NA, Chinn RJ. Imaging of the central nervous system in HIV infection. Imaging 2002;14:48-59.

20. Ruiz A, Post MJ, Bundschu CC. Dentate nuclei involvement in AIDS patients with CNS cryptococcosis: Imaging findings with pathologic correlation. J Comput Assist Tomogr 1997;21:175-82.

21. Saigal G, Post MJ, Lolayekar S, Murtaza A. Unusual presentation of central nervous system Cryptococcal infection in an immunocompetent patient. AJNR Am J Neuroradiol 2005;26:2522-6.

22. Lui G, Lee N, Ip M, Choi KW, Tso YK, Lam E, et al. Cryptococcosis in apparently immunocompetent patients. QJM 2006;99:143-51.

23. Vender JR, Miller DM, Roth T, Nair S, Reboli AC. Intraventricular cryptococeal cysts. AJNR AM J Neuroradiol 1996;17:110-3.

24. Tien RD, Chu PK, Hesselink JR, Duberg A, Wiley C. Intracranial cryptococcosis in immunocompromized patients: CT and MR findings in 29 cases. AJNR Am J Neuroradiol 1991;12:283-9.

25. Chang L, Miller BL, McBride D, Cornford M, Oropilla G, Buchthal S, et al. Brain lesions in patients with AIDS: H-1 MR spectroscopy. Radiology 1995; $197: 525-31$.
26. Wiercinska-Drapalo A, Tarasow E. Magnetic resonance imaging and spectroscopic metabolites assessment in brain cryptococcosis. Wiad Parazytol 2001;47:535-41.

27. Himmelreich U, Dzendrowskyj TE, Allen C, Dowd S, Malik R, Shehan BP, et al. Cryptococcomas distinguished from gliomas with MR spectroscopy: An experimental rat and cell culture study. Radiology 2001;220:122-8.

28. Ho TL, Lee HJ, Lee KW, Chen WL. Diffusion-weighted and conventional magnetic resonance imaging in cerebral cryptococcomas. Acta Radiol 2005;46:411-4.

29. Chan LL, Singh S, Jones D, Diaz EM, Ginsberg LE. Imaging of mucormycosis skull base osteomyelitis. AJNR Am J Neuroradiol 2000;21:828-31

30. Spellberg B, Edwards J Jr, Ibrahim A. Novel perspectives on mucormycosis: Pathophysiology, presentation and management. Clin Microbiol Rev 2005; 18:556-69

31. Rumboldt Z, Castillo M. Indolent intracranial mucormycosis: Case report J Neuroradiol 2002;23:932-4.

32. Horger M, Hebart H, Schimmel H, Vogel M, Brodoefel H, Oechsle K, et al. Disseminated mucormycosis in haematological patients: CT and MRI findings with pathological correlation. Br J Radiol 2006;79:88-95.

33. Terk MR, Underwood DJ, Zee CS, Colletti PM. MR imaging in rhinocerebral and intracranial mucormycosis with CT and pathologic correlation. Magn Reson Imaging 1992;10:81-7.

34. Stave GM, Heimberger T, Kerkering TM. Zygomycosis of the basal ganglia in intravenous drug users. Am J Med 1989;86:115-7.

35. Siegal JA, Cacayorinb ED, Nassif AS, Rizk D, Galambos C, Levy B, et al. Cerebral mucormycosis: Proton MR spectroscopy and MR imaging. Magn Reson Imaging 2000;18:915-20.

36. Tung GA, Rogg JM. Diffusion-weighted imaging of cerebritis. AJNR Am J Neuroradiol 2003;24:1110-3.

37. Mathur S, Karimi A, Mafee MF. Acute optic nerve infarction-demonstrated by diffusion-weighted imaging in a case of rhinocerebral mucormycosis. AJNR Am J Neuroradiol 2007;28:489-90.

38. Bakleh M, Aksamit AJ, Tleyjeh IM, Marshall WF. Successful treatment of cerebral blastomyeosis with voriconazole. Clin Infect Dis 2005;40:6971.

39. Wheat J. Endemic mycoses in AIDS: A Clinical Review. Clin Microbiol Rev 1995;8:146-59

40. Pappas PG. Blastomycosis. Semin Respir Crit Care Med 2004;25:11321

41. Chowfin A, Tight R, Mitchell S. Recurrent blastomycosis of the central nervous system: Case report and review. Clin Infect Dis 2000;30:96971 .

42. Ward BA, Parent AD, Raila F. Indications for the surgical management of central nervous system blastomycosis. Surg Neurol 1995;43:379-88.

43. Witzig RS, Hoadley DJ, Greer DL, Abriola KP, Hernandez RL, Blastomycosis and human immunodeficiency virus: Three new cases and review. South Med J 1994;87:715-9

44. McGahan JP, Graves DS, Palmer PE, Stadalnik RC, Dublin AB. Classic and contemporary Imaging of coccidioidomycosis. A.JR Am J Roentgenol 1981;136:393-404.

45. Hector RF, Laniado-Laborin R. Coccidioidomycosis: A fungal disease of the Americas. PLoS Med 2005;2:e2

46. Arsura EL, Johnson R, Penrose J, Stewart K, Kilgore W, Reddy CM, et al. Neuroimaging as a guide to predict outcomes for patients with coccidioidal meningitis. Clin Infect Dis 2005;40:624-7.

47. Erly WK, Bellon RJ, Seeger JF, Carmody RF. MR imaging of acute coccidioidal meningitis. AJNR Am J Neuroradiol 1999;20:509-14.

48. Erly WK, Labadie E, Williams PL, Lee DM, Carmody RF, Seeger JF. Disseminated coccidioidomycosis complicated by vasculitis: A cause of fatal subarachnoid hemorrhage in two cases. AJNR Am J Neuroradiol 1999;20:1605-8

49. Cortez KJ, Walsh TJ, Bennett JE. Successful treatment of coccidioidal meningitis with voriconazole. Clin Infect Dis 2003;36:1619-22.

50. Wrobel CJ, Meyer S, Johnson RH, Hesselink JR. MR findings in acute and chronic coccidioidomycosis meningitis. AJNR Am J Neuroradiol $1992 ; 13: 1241-5$

51. Hadley MN, Martin NA, Spetzler RF, Johnson PC. Multiple intracranial aneurysms due to Coccidioides immitis infection. Case report. J Neurosurg 1987;66:453-6. 
52. Harris DE, Enterline DS. Neuroimaging of AIDS. I. Fungal infections of the central nervous system. Neuroimaging Clin N Am 1997;7:187-98.

53. Mawhorter SD, Curley GV, Kursh ED, Farver CE. Prostatic and central nervous system histoplasmosis in an immunocompetent host: case report and review of the prostatic histoplasmosis literature. Clin Infect Dis 2000;30:595-8.

54. Bradsher RW. Histoplasmosis and blastomycosis. Clin Infect Dis 1996;22:102-11.

55. Wheat LJ, Musial CE, Jenny-Avital E. Diagnosis and management of central nervous system histoplasmosis. Clin Infect Dis 2005;40:84452.

56. Zalduondo FM, Provenzale JM, Hulette C, Goercki JP. Meningitis, vasculitis and cerebritis caused by CNS histoplasmosis: Radiologicpathologic correlation. AJR Am J Roentgenol 1996;166:194-6.

57. Tan V, Wilkins P, Badve S, Coppen M, Lucas S, Hay R, et al. Histoplasmosis of the central nervous system. J Neurol Neurosurg Psychiatry 1992;55:619-22.

58. Gasparetto EL, Carvalho Neto A, Alberton J, Davaus T, Pianovski MA, Yamauchi E, et al. Histoplasmoma as isolated central nervous system lesion in an immunocompetent patient. Arq Neuropsiquiatr 2005;63:689-92.

59. Hazen KC, Howell SA. Candida, Cryptococcus and other yeasts of medical importance. I n: Murray P, editor. Manual of clinical microbiology. $8^{\text {th }} \mathrm{ed}$. ASM Press: Washington DC; 2003. p.1693-711.

60. Odds FC. Pathogenesis of candida infections. J A Acad Dermatol $1994 ; 31: 2-5$.

61. Richardson MD. Changing patterns and trends in systemic fungal infections. J Antimicrob Chemother 2005;56:5-

62. Smego RA, Perfect JR, Durack DT. Combined therapy with Amphoterincin B and 5- fluorocytocine for candida meningitis. Rev Infect Dis 1984;6:791-801.

63. Lai PH, Lin SM, Pan HB, Yang CF. Disseminated miliary cerebral candidiasis. AJNR Am J Neuroradiol 1997;18:1303-6.

64. Gaviani P, Schwartz RB, Hedley-Whyte ET, Ligon KL, Robicsek A, Schaefer P, et al. Diffusion-weighted imaging of fungal cerebral infection. AJNR Am J Neuroradiol 2005;26:1115-21.

65. Whiteman ML, Bowen BC, Post MJ, Bell MD. Intracranial infections. In: Scott W Atlas, editor. Magnetic resonance imaging of brain and spine. $3^{\text {rd }}$ ed. Lippincott Williams and Wilkins: Philadelphia; 2002.p. 1099-177.

66. Garg M, Gupta RK. MR spectroscopy in intracranial infection. In: Gillard J, Waldman A, Barker P, editors. Clinical MR Neuroimaging. Cambridge University Press: Cambridge; 2005. p. 380-406.

67. Luthra G, Parihar A, Nath K, Jaiswal S, Prasad KN, Husain N, et al. Comparative evaluation of fungal, tubercular and pyogenic brain abscesses with conventional, diffusion MR imaging and proton MR spectroscopy. AJNR Am J Neuroradiol 2007 (in press).

68. Kim CW, Perry A, Currier B, Yaszemski M, Garfin SR. Fungal infections of the spine. Clin Orthop Relat Res 2006;444:92-9.

69. Jinkins JR. Magnetic resonance imaging of spinal infectious disease:
Pathophysiologic concepts of origin and spread. Acta Clin Croat 2002;41:29-30.

70. Mahboubi S, Morris MC. Imaging of spinal infections in children. Radiol Clin North Am 2001;39:215-22.

71. Williams RL, Fukui MB, Meltzer CC, Swarnkar A, Johnson DW, Welch W. Fungal spinal osteomyelits in the immunocompromized patient: MR findings in three cases. AJNR Am J Neuroradiol 1999;20:381-5.

72. Saigal G, Donovan Post MJ, Kozic D. Thoracic intradural Aspergillus abscess formation following epidural steroid injection. AJNR Am J Neuroradiol 2004;25:642-4.

73. Zeppa MA, Laorr A, Greenspan A, McGahan JP, Steinbach LS. Skeletal coccidioidomycosis: Imaging findings in 19 patients. Skeletal Radiol $1996 ; 25: 337-43$.

74. Olson EM, Duberg AC, Herron LD, Kissel P, Smilovitz D. Coceidioidal spondylitis: MR findings in 15 patients. AJR Am J Roentgenol 1998;171:785-9.

75. Grosse P, Tintelnot K, Söllner O, Schmitz B. Encephalomyelitis due to Cryptococcus neoformans var gattii presenting as spinal tumor: Case report and review of the literature.. J Neurol Neurosurg Psychiatry 2001;70:113-6.

76. Lie KW, Yu YL, Cheng IK, Woo E, Wong WT. Cryptococeal infection of the lumbar spine. J R Soc Med 1989;82:172-3.

77. Deus-Silva L, Costa AE, Bevilacqua JM, Assis DB, Ferraz CA Jr, Oliveira AC, et al. Meningoradiculitis due to Cryptococeus neoformans in an immunocompetent patient. Arq Neuropsiquiatr 2004;62:147-9.

78. Gupta SK, Chhabra R, Sharma BS, Das A, Khosla VK. Vertebral cryptococesis simulating tuberculosis. Br J Neurosurg 2003;17:5569

79. Chhem RK, Wang S, Jaovisidha S, Schmit P, Friedman L, Bureau NJ, et al. Imaging of fungal, viral and parasitic musculoskeletal and spinal diseases. Radiol Clin North Am 2001;39:357-78.

80. Aganovic L, Hoda RS, Rumboldt Z. Hyperintensity of spinal Cryptococcus infection on diffusion-weighted MR Images. AJR Am J Roentgenol 2004;183:1176-7.

81. Saccente M, Abernathy RS, Pappas PG, Shah HR, Bradsher RW. Vertebral blastomycosis with paravertebral abscess: Report of eight cases and review of the literature. Clin Infect Dis 1998;26:413-8.

82. Hardjasudarma M, Willis B, Black-Payne C, Edwards R. Pediatric spinal blastomycosis: Case report. Neurosurgery 1995;37:534-6.

83. Parr AM, Fewer D. Intramedullary blastomycosis in a child: Case report. Can J Neurol Sci 2004;31:282-5.

84. Frazier DD, Campbell DR, Garvey TA, Wiesel S, Bohlman HH, Eismont FJ. Fungal infections of the spine: Report of eleven patients with longterm follow-up. J Bone Joint Surg Am 2001;83:560-5.

Accepted on 26-05-2007

Source of Support: Nil, Conflict of Interest: None declared. 